\title{
Computed tomography findings of focal lung lesion and their pathological correlations
}

\section{Sharma S', Pant HP2, Pandit SP3}

'Saroj Sharma, Consultant Radiologist; ${ }^{2} \mathrm{Hom}$ Prasad Pant, MD Resident; ${ }^{3}$ Swoyam Prakash Pandit, Associate Professor, National Academy of Medical Sciences, Kathmandu, Nepal

\begin{abstract}
Background: Focal lung masses are common radiological findings which need to be characterized to plan management. Suspicious lesions require invasive investigations and aggressive treatment. However, likely benign lesions require no invasive investigations.

Objectives: We aimed to correlate the computerized tomography findings of focal lung lesions with pathological diagnoses.

Methods: A total of 56 patients were enrolled in the study. Plain and contrast enhanced computerized tomography scan of chest was done in spiral computerized tomography. Radiologist's computerized tomography reports were obtained. Pathological diagnosis were followed in all cases and recorded. computerized tomography reports were compared with pathological reports and validity of computerized tomography in predicting the nature of lung lesion was assessed.

Results: Overall, squamous cell carcinoma was the most common histological cell type (46.34\%) followed by adenocarcinoma (41.46\%). In $95.12 \%$ of total cases, size of the malignant lesion was more than $3 \mathrm{~cm}$. Squamous cell carcinoma was the most common tumor located centrally (71.42\%) and adenocarcinoma peripherally (55.55\%). Spiculated margin was most commonly associated in malignant lesions. Among morphological characters, chest wall invasion and mediastinal invasion were statistically significant for malignancy. Contrast enhancement more than 20 hounsfield unit had more than $80 \%$ sensitivity and specificity for malignant lesions. Sensitivity of computerized tomography combining all morphological characteristics for depicting malignancy was $97.6 \%$ and specificity was $46.7 \%$.

Conclusion: The present study showed that contrast enhanced computerized tomography provides more precise tumor localization, characterization and intrathoracic extension to differentiate benign from malignant lung lesion. Enhancement of the lesion is more specific for malignancy than other morphological characteristics.
\end{abstract}

Key words: Computerized Tomography scan, Lung mass, Malignancy

\section{INTRODUCTION}

H ocal lesion is an infection, tumor, or injury that develops at a restricted or circumscribed area of tissue ${ }^{1}$. Focal pulmonary lesions (FPL) are among the most common findings in radiology practice. At imaging, disease may present as nodular opacity or localized area of consolidation. It includes solitary pulmonary nodule (SPN) and masses. SPN is an approximately round lesion that is less than $3 \mathrm{~cm}$ in diameter and is completely surrounded by pulmonary parenchyma, without other abnormalities ${ }^{2}$. Lesions larger than $3 \mathrm{~cm}$ are called masses and are often malignant. Causes of such FPL include malignant lesions such as bronchogenic

Address for correspondence

Dr. Hom Prasad Pant

MD Resisdent, National Academy of Medical Sciences

Kathmandu, Nepal

E-mail: homprasadpant@yahoo.com carcinoma, solitary metastases and carcinoid tumors; and benign tumors such as granuloma, hamartoma, vascular processes and focal areas of inflammation. Bronchogenic carcinoma, a malignant neoplasm with an alveolar or bronchial origin in over $99.5 \%$ of cases, is the most common cancer and cause of cancer-related death in both men and women. In Nepal, lung cancer is on the rise. It is estimated that 8,000 to 10,000 cases of lung cancer are diagnosed each year and almost $90 \%$ of them die the same year ${ }^{3}$.

Modern imaging techniques are of paramount importance in the detection and classification of focal abnormalities of the lung parenchyma. One of the most challenging tasks for a radiologist in evaluation of focal lung lesions is to classify an abnormality as benign or malignant. Because a focal lung lesion is one of the most frequent presentations of bronchogenic carcinoma, 
patients with such lesions need to be identified in order to allow for an aggressive and potentially invasive approach to establish the exact diagnosis. In contrast, lesions that display features of benign disease may be subjected to a more conservative management and imaging findings may obviate the need for biopsy or thoracotomy in such cases².

There are multiple ways of radiological evaluation including conventional chest $x$-ray, ultrasonography, CT (computed tomography) scan, magnetic resonance imaging (MRI), and positron emission tomography (PET). Biopsy is the only confirmatory investigation to differentiate benign from malignant. However, most of the time biopsy is not warranted due to its invasive nature. Chest CT is routinely available cost effective investigation modality too. It supplements routine chest $\mathrm{X}$-ray by providing better visualization of the nodule; improving sensitivity for calcification and multiple lesions and guiding transthoracic needle aspiration and biopsy. In addition to providing precise characterization of size, contour, extent and tissue comparison of suspicious lesion, it serves as a part of staging process to assess the extent of disease. In this study, we aimed to evaluate and analyze the $\mathrm{CT}$ findings, and compare $\mathrm{CT}$ findings with pathological diagnosis considering the pathology as gold standard in diagnosis ${ }^{4}$.

\section{METHODS}

This study was carried out in the Department of Radiology, Bir hospital and NAMS affiliated hospitals. Ethical clearance was taken from the respective institutions. Informed written consent from each patient was taken for this study. This was hospital based study done during three years from November 2010 to October 2013. Patients referred to the radiology department from age 15 years and above for CT scan of chest with suspicious lung lesion were included in the study. A total of 56 patients with FPL were enrolled in study. Patients with multiple lesions, known primary elsewhere or in lung and patient with contraindication for invasive procedure (biopsy) were excluded.

Subjects of the study underwent CT scan in SHIMADZU Spiral CT machine. Seven millimetre cut section with $5 \mathrm{~mm}$ sections at the level of lesion both before and after intravenous contrast enhancement was obtained. Sections were obtained from the lung apices to diaphragm routinely including the adrenals. About 75 to $100 \mathrm{ml}$ of non-ionic water soluble contrast media (Ultravist or Optiray) of strength $300 \mathrm{mgl} / \mathrm{ml}$ was used for enhancement. CT scan images were obtained in lung, mediastinal and bone window.
$\mathrm{CT}$ reports were obtained. Reports fulfilling the inclusion criteria were recorded in data collection sheet. Patients were advised for pathological diagnosis. USG or CT guided or bronchoscopic fine needle aspiration cytology (FNAC) were done under the supervision of radiologist. For centrally located lesions, subjects were advised to undergo fibreoptic bronchoscopic biopsy. Finally pathological reports were collected and recorded. CT reports were compared with pathological reports and validity of $\mathrm{CT}$ in predicting the nature of lung lesion was assessed.

Data was entered and analyzed in Statistical Package for Social Sciences (SPSS) 17.0 program. Chi Square test and Fischer's Exact test were used for statistical analysis.

\section{RESULTS}

Out of the 56 patients, males were more in number (Figure 1). Male to female ratio in our study was 2.5:1.

Among the 40 male patients, 28 had malignant and 12 had benign lesions. Out of the 16 female patients, malignant lesion was found in 13 and benign in three patients. So the male to female ratio for malignancy was 2.15:1. The minimum age of patient in our study was 21 years and the maximum was 79 years. The mean age was 57.52 years. The largest group of patients was in the range of 55 to 64 years. Minimum age for malignancy was 21 years in female with soft tissue sarcoma and 27 years in male with squamous cell carcinoma.

Among 56 patients, 43 were smokers and 13 were nonsmokers. Out of 41 patients with malignancy $36(87.80 \%)$ were smokers and $5(12.19 \%)$ were non-smokers (figure 2).

Distribution of the lesion in the right and left lungs were almost equal. But the predominance of the upper lobe can be seen for both benign and malignant lesions. Out of 43 smokers, 17 (39.53\%) had squamous cell carcinoma, $15(34.88 \%)$ had adenocarcinoma, 7 (16.27\%) had benign lesions, 3 (6.97\%) had small cell carcinoma and 1 (2.32\%) had bronchoalveolar carcinoma. Among the 13 nonsmokers, 8 (61.5\%) had benign lesion, $2(15.38 \%)$ had squamous cell carcinoma, 2 (15.38\%) adenocarcinoma and $1(7.69 \%)$ had soft tissue sarcoma.

Out of the 56 cases, 15 (26.78\%) were proven to be benign and 41 (73.22\%) malignant on pathology. Among the malignant lesions, most common was squamous cell carcinoma 19/41 (46.34\%) followed by adenocarcinoma which comprised $17(41.46 \%)$ of the total malignant cases. Small cell carcinoma, bronchoalveolar carcinoma 
and soft tissue sarcoma accounted for $7.31 \%, 2.43 \%$ and $2.43 \%$ respectively.

Among the total 56 patients, $46(82.14 \%)$ had lesions $>3 \mathrm{~cm}$ and $10(17.85 \%)$ had lesions $\leq 3 \mathrm{~cm}$. Of the total 41 malignant cases, 39 (95.12\%) of cases were more than three $\mathrm{cm}$ in size and $2(4.88 \%)$ were less or equal to $3 \mathrm{~cm}$. Of the two lesions of size less than $3 \mathrm{~cm}$ size, all were squamous cell carcinoma (Table 1).

Out of the total 56 patients, $37(66.07 \%)$ had peripheral lesion and 19 (33.92\%) had central lesion. Of the malignant lesions, 27 (65.85\%) were located in periphery and 14 (34.14\%) were located centrally. Out of 27 peripherally located malignant lesions $15(55.55 \%)$ were adenocarcinoma and 9 (33.33\%) were squamous cell carcinoma. Among 14 centrally located malignancies, $10(71.42 \%)$ were squamous cell carcinoma and 2 (14.28\%) were adenocarcinoma. It was found that adenocarcinoma was more common in peripheral and squamous cell carcinoma in central location.

Out of the 41 malignant lesions, margins were spiculated in $16(39.02 \%)$, smooth in $12(29.26 \%)$, irregular in 7 $(17.0 \%)$ and lobulated in $6(14.63 \%)$ patients. Among benign lesions, 10 (66.66\%) had smooth, 3(20\%) had spiculated, 1 (6.66\%)had irregular and 1(6.66\%) had lobulated margin. Statistical analysis using chi square test showed that the type of margin was statistically significant in CT interpretation $(p=0.026)$ and on pathological interpretation it was statistically insignificant ( $p$-value $=0.091$ ). Calcification was absent in $48(85.71 \%)$ cases. Out of eight remaining cases, $6(10.71 \%)$ malignant and $2(3.57 \%)$ benign lesions had calcification. Among six malignant lesions with calcification, four were squamous cell carcinoma and two were adenocarcinoma. In our study, the majority of the lesions showed punctuate and amorphous pattern of calcification. Out of six malignant calcified lesions 4 $(66.66 \%)$ showed punctate type of calcification.

Out of the 56 cases of our study, cavitations were absent in $26(46.42 \%)$ cases. Of the remaining 30 cases, it was present in $26(46.42 \%)$ malignant and $4(7.14 \%)$ benign cases. Among 26 cavitatory malignant lesions, 18 (69.23\%) had eccentric location and $8(30.76 \%)$ had central location. Cavitation was more common in squamous cell carcinoma, 14 out of 26 (53.84\%), followed by adenocarcinoma. Chest wall and mediastinal invasion were almost always noted in malignant lesion. Other associated morphological characteristics were also more common in malignant compared with benign focal lesions.

Out of the 41 pathologically proven malignant cases, $36(87.80 \%)$ showed enhancement $>20 \mathrm{HU}$ and only 5 (12.19\%) cases had enhancement $\leq 20 \mathrm{HU}$. Meanwhile, most of the benign lesions had enhancement $\leq 20 \mathrm{HU}$ (Table 3). The sensitivity of CT enhancement to predict malignancy was $87.8 \%$, and specificity was $80.0 \%$. The positive predictive value was $92.3 \%$ and negative predictive value was $70.6 \%$. Statistical analysis showed the findings to be significant ( $p$-value $=0.001$ ).

Table 1: Pathological diagnosis of cases

\begin{tabular}{cccccccc}
\hline & \multicolumn{5}{c}{ Histology } \\
Benign & $\begin{array}{c}\text { Malignant } \\
\text { Squamous cell } \\
\text { carcinoma }\end{array}$ & $\begin{array}{c}\text { Adeno } \\
\text { carcinoma }\end{array}$ & $\begin{array}{c}\text { Small cell } \\
\text { carcinoma }\end{array}$ & $\begin{array}{c}\text { Basal cell } \\
\text { carcinoma }\end{array}$ & $\begin{array}{c}\text { Soft tissue } \\
\text { sarcoma }\end{array}$ & Total \\
15 & 19 & 17 & 3 & 1 & 1 & 56 \\
$(26.78 \%)$ & $(33.92 \%)$ & $(30.35 \%)$ & $(5.35 \%)$ & $(1.78 \%)$ & $(1.78 \%)$ & $(100 \%)$ \\
\hline
\end{tabular}

Table 2: Correlation of CT findings with the pathological results

\begin{tabular}{|c|c|c|c|c|c|c|c|c|}
\hline \multirow{3}{*}{\multicolumn{2}{|c|}{ Size $(\mathrm{cm})$}} & & \multicolumn{4}{|c|}{ Pathological Interpretation } & \multirow{2}{*}{\multicolumn{2}{|c|}{ Total }} \\
\hline & & & \multicolumn{2}{|c|}{ Benign } & \multicolumn{2}{|c|}{ Malignant } & & \\
\hline & & & $\mathbf{N}$ & $\%$ & $\mathbf{N}$ & $\%$ & $\mathbf{N}$ & $\%$ \\
\hline \multirow{3}{*}{$\leq 3$} & \multirow{3}{*}{ CT Interpretation } & Benign & 6 & $100.0 \%$ & 0 & $0.0 \%$ & 6 & $100.0 \%$ \\
\hline & & Malignant & 2 & $50.0 \%$ & 2 & $50.0 \%$ & 4 & $100.0 \%$ \\
\hline & & Total & 8 & $80.0 \%$ & 2 & $20.0 \%$ & 10 & $100.0 \%$ \\
\hline \multirow{3}{*}{$>3$} & \multirow{3}{*}{ CT Interpretation } & Benign & 1 & $50.0 \%$ & 1 & $50.0 \%$ & 2 & $100.0 \%$ \\
\hline & & Malignant & 6 & $13.6 \%$ & 38 & $86.4 \%$ & 44 & $100.0 \%$ \\
\hline & & Total & 7 & $15.2 \%$ & 39 & $84.8 \%$ & 46 & $100.0 \%$ \\
\hline
\end{tabular}


Table 3: Type of lesion by enhancement pattern

\begin{tabular}{|c|c|c|c|c|c|c|c|c|}
\hline \multirow{2}{*}{\multicolumn{3}{|c|}{ Enhancement pattern difference (HU) }} & \multicolumn{4}{|c|}{ Path Interpretation } & \multirow{2}{*}{\multicolumn{2}{|c|}{ Total }} \\
\hline & & & \multicolumn{2}{|c|}{ Benign } & \multicolumn{2}{|c|}{ Malignant } & & \\
\hline & & & $\mathbf{N}$ & $\%$ & $\mathbf{N}$ & $\%$ & $\mathbf{N}$ & $\%$ \\
\hline \multirow{3}{*}{$\leq 20$} & \multirow{3}{*}{$\mathrm{CT}$ Interpretation } & Benign & 6 & $85.7 \%$ & 1 & $14.3 \%$ & 7 & $100.0 \%$ \\
\hline & & Malignant & 6 & $60.0 \%$ & 4 & $40.0 \%$ & 10 & $100.0 \%$ \\
\hline & & Total & 12 & $70.6 \%$ & 5 & $29.4 \%$ & 17 & $100.0 \%$ \\
\hline \multirow{3}{*}{$>20$} & & Benign & 1 & $100.0 \%$ & 0 & $0.0 \%$ & 1 & $100.0 \%$ \\
\hline & CT Interpretation & Malignant & 2 & $5.3 \%$ & 36 & $94.7 \%$ & 38 & $100.0 \%$ \\
\hline & & Total & 3 & $7.7 \%$ & 36 & $92.3 \%$ & 39 & $100.0 \%$ \\
\hline
\end{tabular}

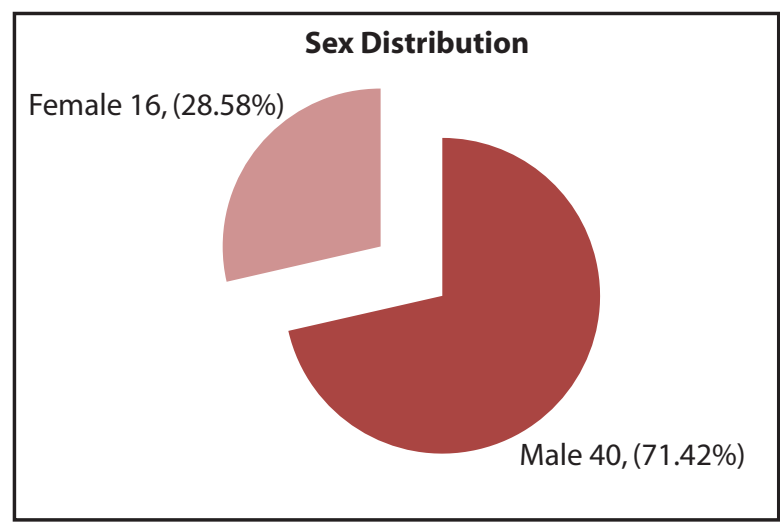

Figure 1: Sex distribution of the cases

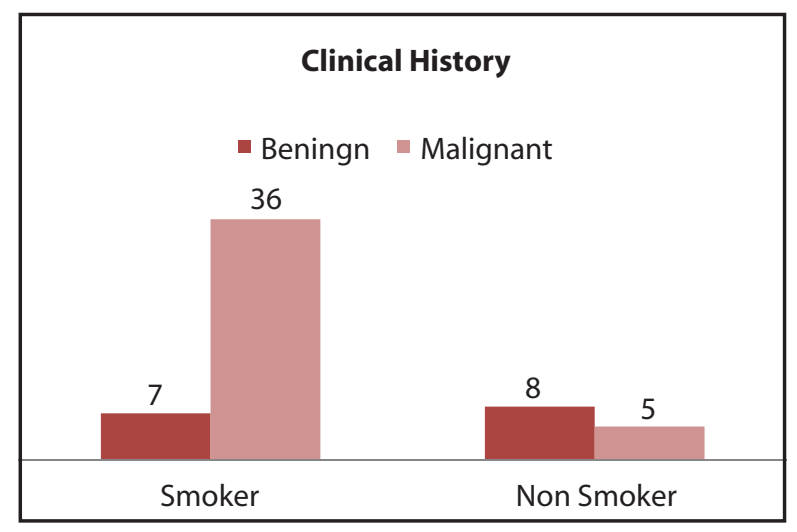

Figure 2: Clinical history of smoking and malignancy status

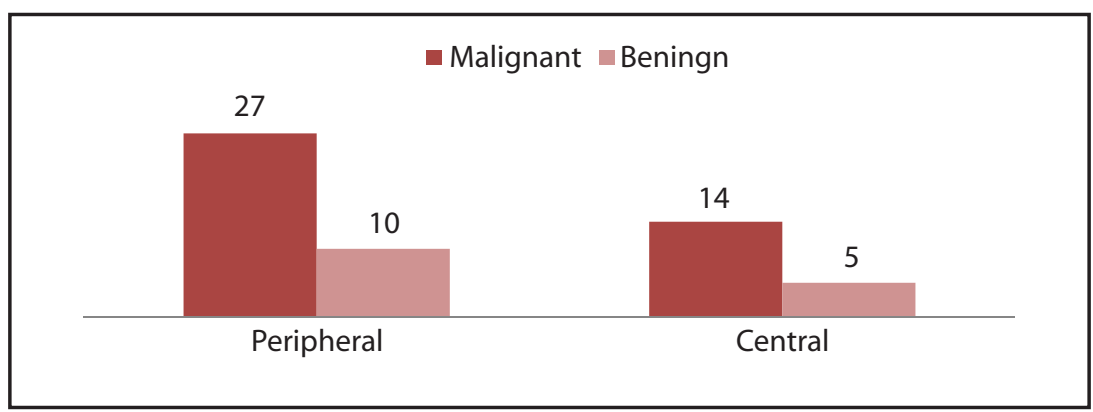

Figure 3: Type of lesion by central and peripheral location

Of the 48 cases that were diagnosed as malignant by $\mathrm{CT}, 40$ were proven malignant by histology and eight of them were benign. Out of eight cases interpreted as benign by $\mathrm{CT}$, seven cases were proved benign and 1 malignant. Thus $\mathrm{CT}$ prediction of malignancy by combining all CT characteristics had a sensitivity of $97.6 \%$, a specificity of $46.7 \%$, a positive predictive value of $83.3 \%$ and a negative predictive value of NPV $87.5 \%$.

\section{DISCUSSION}

This study was done to evaluate morphological characters of benign and malignant lung lesions by plain and contrast CT scan and to correlate the radiological impression with pathological report. Incidence of lung mass by Burns J study shows equal male to female ratio $^{5}$. Some of the studies have uniformly reported a male predominance ${ }^{6}$. Our study showed similar result, male: female; 2.15:1. In the present study, the highest incidence of lung lesion was noted in the age range of 55-64 years with mean age of 57.52 years which is similar to various other studies. Out of total malignant lesions, 3 (7.31\%) patients were under 30 years of age. This shows that lung malignancy can occur even below 30 years of age. Malignancy was highly associated with smoking (87.80\%). Out of total 36 smokers with malignancy, most common cell type in our study was squamous cell carcinoma $(47.22 \%)$ followed by adenocarcinoma (41.665). This is similar to that of Chhajed $^{6}$ who also found squamous cell carcinoma to be the most common cell type in smokers followed 
by adenocarcinoma. It was seen that smoking is most commonly associated with squamous cell carcinoma. In our study, among non smokers both squamous and adenocarcinoma had equal incidence which is against Chhajed $^{6}$ where adenocarcinoma was common among non smokers. These findings show that smoking has high association with lung carcinoma. In our study, risk of having malignancy in smokers is 7.2 times higher than in non-smokers which is similar to many studies. Right lungs were involved in $53.57 \%$ of cases and left lung in $46.42 \%$. Sixty two percent of the total lesions were located in the upper lobe distributed almost equally on both sides. In the Shetty CM study, right lung was involved in 44\%, left in $36 \%$ and both lungs in twenty percent ${ }^{7}$. They also found upper lobe predominance. Of the total malignant lesions, 58.53\% were located in the upper lobe which was almost similar to Shah PK $(72 \%)^{8}$. Most $(82.14 \%)$ of the lesions were $>3 \mathrm{~cm}$ and $17.85 \%$ were $\leq 3 \mathrm{~cm}$. Of the total malignant cases, $95.12 \%$ were more than $3 \mathrm{~cm}$ in size and $4.88 \%$ were less or equal to $3 \mathrm{~cm}$. These findings were similar to Hollings $\mathrm{N}$ et al who showed lesions $>3 \mathrm{~cm}$ were associated with malignancy in 93-99\% of cases ${ }^{9}$. Similarly Tang AWK ${ }^{42}$ found association of malignancy in lesion less than $1 \mathrm{~cm}$ in $8 \%$ of cases which was also seen in our study. Thus no reliable conclusion can be based on size criteria alone as small nodules can be malignant and larger ones benign.

Most common histological type noted in our study was squamous cell carcinoma accounting for $46.34 \%$ of cases followed by adenocarcinoma which account for $41.46 \%$. The findings were similar to most other studies which found squamous cell carcinoma the most common type. However the study done by Chhajed $^{6}$ reported $^{2}$ adenocarcinoma as the most common type. In this study, peripheral lesions were more frequent (66.07\%) than central ones (33.9\%) which was similar to study by Yeon PJ et al but different from other study ${ }^{10}$.

High number of peripheral lesion in our study is due to easy FNAC/Biopsy of peripheral lesions under image guidance than central lesions. Among peripheral malignant lesions adenocarcinoma was the most common accounting for (55.55\%) and among central malignant lesion squamous cell carcinoma (71.42\%) was more common. Out of 27 peripherally located malignant lesions 15 (55.55\%) were adenocarcinoma and 9(33.33\%) were squamous cell carcinoma. Among 14 centrally located malignancies 10 (71.42\%) were squamous cell carcinoma and 2 (14.28\%) were adenocarcinoma. It was found that inspite of large number of peripherally located malignant lesions, squamous cell carcinoma was most common due to large number of squamous cell carcinoma than adenocarcinoma among central lesions.

In the present study, 39.02\% of malignant lesions showed spiculated margins and $29.26 \%$ showed smooth margin. Irregular margin was seen in $17 \%$ and lobulated margin in $14.63 \%$. It shows that smooth margin can also be present in malignant lesion which was also shown by Marten and Erasmus findings. Thus in conclusion spiculated margin was highly suggestive of malignancies as shown in present and different other studies but smooth margin cannot rule out malignancy. Calcification was seen in $14.29 \%$ of lesions which is in concordance with other studies. Among malignant lesion with calcification, squamous cell carcinoma was the most common (66.66\%). This is similar to that reported by Grewal ${ }^{11}$. Punctate type of calcification was the most common. Calcification was more common in peripherally located tumors than in centrally located ones.

Out of 56 cases in our study, cavitation was absent in $26(46.42 \%)$ of cases. Of the remaining 30 cases, it was present in 26 (46.42\%) malignant and 4 (7.14\%) benign cases. Among 26 cavitatory malignant lesions, 18 (69.23\%) had eccentric location and 8 (30.76\%) had central location. Cavitation was more common in squamous cell carcinoma, 14 out of 26 (53.84\%), followed by adenocarcinoma. This correlates with study done by Sharmaet al who also reported cavitation to be seen most commonly in squamous cell carcinoma $(67 \%)^{12}$. In our study, more than $20 \mathrm{HU}$ of enhancement of lung lesion after IV contrast was highly suggestive of malignancy, which is similar to other studies. When reducing the enhancement threshold to $<=15 \mathrm{HU}$, sensitivity was $98 \%$ and specificity was $58 \%$ shown in Swenson study in $2000^{13}$. In our study, the sensitivity of $\mathrm{CT}$ scan to predict malignancy by combining all morphological characters was $97.6 \%$ and specificity was $46.7 \%$ which is comparable with $\mathrm{Yi} \mathrm{CA}^{14}$ and Seemann $\mathrm{MD}^{15}$ study.

\section{CONCLUSION}

From our study, we conclude that squamous cell carcinoma is the most common histological cell type and is also most common among smokers in our context too. Most of the centrally located malignant lesions are more likely to be squamous cell carcinoma and peripherally located- adenocarcinoma. Malignant lesion present mostly with spiculated margin. We found that compared to all morphological characters the enhancement pattern is most sensitive and specific to predict malignancy. Sensitivity of CT combining all morphological characteristics is high (97.6\%) but 
specificity is low (46.7\%). Specificity of enhancement (80\%) age rather found to be much better than combining all morphological characters.

\section{REFERENCES}

1. Mosby's Medical Dictionary, 4th ed. St. Louis: Mosby Elsevier; 1994. Focal Lesion; p. 629

2. Ost D, Fein AM and Feinsilver SH. The solitary pulmonary nodule. The new England Journal of Medicine. 2003; 384: 2535-42

3. Piya MK, Acharya SC. Oncology in Nepal. South Asian Journal of Cancer. 2012;1(1):5-8.

4. Edward F, Patz Jr. Imaging Bronchogenic Carcinoma. Chest 2000 April; 117:90-5.

5. Burns J, Haramati LB, Whitney K, Zelefsky MN. Consistency of reporting basic characteristics of lung nodules and masses on computed tomography. AcadRadiol.; 2004 Feb;11(2):233-7.

6. Chhajed PN, Athavale AU, Shah AC. Clinical and Pathological Profile of 73 Patients with lung cancer:Is the Picture changing? J Assoc Physicians India. 1999 May;47(5):483-87.

7. Shetty CN, Lakhkar BM, Gangadhar VSS and Ramachandran NR. Changing pattern of bronchogenic carcinoma. Indian Journal of radiology and imaging. 2005; 15:233-238.

8. Shah PK, Austin JHM, White CS, Patel P,Haramati LB, Pearson GDN et.al. Missed Non-Small Cell Lung Cancer: Radiographic Findings of Potentially Resectable Lesions Evident Only in Retrospect. Radiology. 2003 Jan; 226: 235-41.
However, in our context, further study in large population is recommended before they can be practically implemented.

9. Hollings N, Shaw P. Diagnostic Imaging of lung cancer. European Respiratory Journal. 2002 April; 19(4):722-42.

10. Yeon PJ, Hyun DK, Seong LJ, Soo KM. Characteristic radiological findings of adenocarcinoma with micro papillary pattern, subtype of the lung. Journal of thoracic oncology; 2007; 2:573-4.

11. GrewalRG, Austin JH. CT demonstration of calcification in carcinoma of the lung. J Comput Assist Tomography 1994 Nov-Dec; 18(6):867-71.

12. Sharma CP, Bchera D, Aggarwal AN, Gupta D and Jindal SK.Radiographic Pattern in Lung Cancer.Indian Journal of chest Disease and Allied Sciences.2002;44:25-30.

13. Swensen SJ, Viggiano RW, Midthun DE, et al. Lung nodule enhancement at CT: multicenter study. Radiology 2000;214:73-80.

14. Yi CA, Lee KS, Kim EA, et al. Solitary pulmonary nodules: dynamic enhanced multi-detector row CT study and comparison with vascular endothelial growth factor and microvessel density. Radiology 2004;233:191-99.

15. Seemann MD, Staebler A, Beinert T, Dienemann $H$, Obst B, Matjko M et al. Usefulness of Morphological Characteristics for the differentiation of benign from malignant Solitary Pulmonary Lesion using HRCT. European Radiology. 1999; 9(3):409-17. 